\title{
Finite-Temperature Behavior of an Inter-species Fermionic Superfluid with Population Imbalance
}

\author{
Hao Guo ${ }^{1}$, Chih-Chun Chien ${ }^{1}$, Qijin Chen ${ }^{2,1}$, Yan $\mathrm{He}^{1}$, and K. Levin ${ }^{1}$ \\ ${ }^{1}$ James Franck Institute and Department of Physics, University of Chicago, Chicago, Illinois 60637, USA and \\ ${ }^{2}$ Zhejiang Institute of Modern Physics and Department of Physics, \\ Zhejiang University, Hangzhou, Zhejiang 310027, China
}

(Dated: October 10, 2018)

\begin{abstract}
We determine the superfluid transition temperature $T_{c}$ and related finite temperature phase diagrams for the entire BCS-Bose Einstein condensation crossover in a homogeneous mixture of ${ }^{6} \mathrm{Li}$ and ${ }^{40} \mathrm{~K}$ atoms with population imbalance. Our work is motivated by the recent observation of an inter-species Feshbach resonance. Pairing fluctuation effects, which significantly reduce $T_{c}$ from the onset temperature for pairing $\left(T^{*}\right)$, provide reasonable estimates of $T_{c}$ and indicate that the inter-species superfluid phase should be accessible in future experiments. Although a generalized-Sarma phase is not stable in the ground state near unitarity, our phase diagrams show that it appears as an intermediate-temperature superfluid.

PACS numbers: 03.75.Ss, 03.75.Hh,05.30.Fk
\end{abstract}

Ultracold Fermi gases with tunable attractive interactions provide an exciting opportunity to study superfluidity in the very general context of a crossover from BCS theory to BoseEinstein condensation (BEC). While initial experiments addressed the more conventional situation of intra-species pairing with equal populations of the two effective spin states, more recently there has been an emphasis on population imbalanced gases [1, 2, 3, 4, 5]. Many interesting phases have been contemplated including two which appear to have been observed in the laboratory: the so-called Sarma phase [6, 7] which represents a homogeneously polarized state, and a phase separated (or inhomogeneously polarized) state [8].

Adding to the excitement is the possibility of discovering a new form of superfluid involving inter-species pairing. A first step en route to the discovery is the recent observation of Feshbach resonances between ${ }^{6} \mathrm{Li}$ and ${ }^{40} \mathrm{~K}$ atoms [9]. If the transition temperatures are accessible, this tunable attractive interaction should enable BCS-BEC crossover in superfluid phases associated with unequal mass pairing. Moreover, it is of particular interest for its relevance to color superconductivity in quark matter [10].

In this paper we determine the transition temperatures for the entire BCS-BEC crossover in a homogeneous mixture of ${ }^{6} \mathrm{Li}$ and ${ }^{40} \mathrm{~K}$ atoms with population imbalance. In addition we address the temperature-polarization phase diagrams associated with inter-species superfluid phases at and around unitarity. We consider only a homogeneously polarized superfluid and exclude from consideration the phase separated state principally because it is now clear [5] that the normal regions in this heterogeneous phase correspond to a complicated correlated normal state, which is currently difficult to characterize at an analytical level, except in the limit of extreme population imbalance. This normal state, which is distinct from a free Fermi gas, has been addressed numerically using quantum Monte Carlo simulations [11].

Our $T \neq 0$ calculations are performed in a fashion consistent with a generalized BCS-Leggett ground state which has been studied previously [12, 13] in the strict $T=0$ limit. These calculations show the importance of including pairing fluctuations which greatly suppress the transition temperature $T_{c}$ from the pair formation temperature $T^{*}$. An understanding of finite temperature effects positions us to address actual experiments (which are never in the ground state). Moreover, new superfluid phases appear which are constrained to an intermediate regime of non-zero temperatures. That is, they are associated with a lower as well as upper critical temperature. We show that, in the absence of a trap, this intermediate temperature superfluid will be extremely difficult to observe when the heavy species is the majority, but it should be more accessible for the case where the heavy species is the minority. Finally, we study how the phase diagram evolves as one crosses from BCS to BEC. In contrast to polarized Fermi gases with equal mass, close to, but on the BEC side of resonance, the intermediate temperature superfluid disappears when the lighter species is the majority, giving way to a conventional polarized superfluid with only one transition temperature.

There have been extensive studies on zero temperature properties of homogeneous [12] as well as trapped interspecies Fermi gases [12, 14, 15]. This body of work (like that in the present paper) is based on a natural generalization of the BCS-Leggett ground state to accommodate unequal populations. Similarly, while a two channel model formalism may be more relevant to the narrow resonances seen in Ref. [9], all work to date (including our own) deals with the simpler one channel model. Theoretical studies at finite temperatures that are consistent with these $T=0$ calculations has been limited to a strict mean field approach [13] which ignores the important effects of pair fluctuations, or non-condensed pairs.

We begin with an outline of the central equations associated with a generalized "Sarma" state appropriate to the case of unequal masses and finite polarization. This is followed by a short microscopic derivation. We choose the convention $m_{\downarrow}>m_{\uparrow}$, so that the mass of spin-down fermions is the larger. The mass ratio is $m_{\downarrow} / m_{\uparrow}=6.7$. We define $E_{k \uparrow, \downarrow}=$ 
$E_{k} \pm \xi_{k}^{-}, E_{k}=\sqrt{\xi_{k}^{+2}+\Delta^{2}}$, where $\xi_{k}^{ \pm}=\left(\xi_{k \uparrow} \pm \xi_{k \downarrow}\right) / 2$. Here $\xi_{k \sigma}=\epsilon_{k \sigma}-\mu_{\sigma}$, and $\epsilon_{k \sigma}=k^{2} / 2 m_{\sigma}$, where $\sigma=\uparrow, \downarrow$. The four unknowns which must be determined at general temperature $T$ involve the two fermionic chemical potentials $\mu_{\uparrow}$, $\mu_{\downarrow}$, and the excitation gap $\Delta$, whose square appears in $E_{k}$. The quantity $\Delta^{2}$ contains a contribution from condensed (sc) and non-condensed (pg) pairs:

$$
\Delta^{2}=\Delta_{s c}^{2}+\Delta_{p g}^{2}
$$

so that one of the gap components $\left(\Delta_{p g}\right)$ must be separately determined in order to establish the transition temperature $T_{c}$. This is the lowest temperature at which $\Delta_{s c}$ vanishes.

There are then four equations, three of which can be associated with strict mean field theory, and all of which are derived microscopically below. The equations for the total number $n=n_{\uparrow}+n_{\downarrow}$ and number difference $\delta n=n_{\downarrow}-n_{\uparrow}$ of fermions are

$$
n=\sum_{\mathbf{k}}\left\{\left(1-\frac{\xi_{k}^{+}}{E_{k}}\right)+\left[f\left(E_{k \uparrow}\right)+f\left(E_{k \downarrow}\right)\right] \frac{\xi_{k}^{+}}{E_{k}}\right\}
$$

and

$$
\delta n=\sum_{\mathbf{k}}\left[f\left(E_{k \downarrow}\right)-f\left(E_{k \uparrow}\right)\right] .
$$

Here $f(x)=\left(e^{x / T}+1\right)^{-1}$ is the Fermi distribution function. The gap parameter $\Delta$ is obtained from

$$
-\frac{M}{2 \pi a}=\sum_{\mathbf{k}}\left[\frac{1-f\left(E_{k \uparrow}\right)-f\left(E_{k \downarrow}\right)}{2 E_{k}}-\frac{1}{\epsilon_{k}}\right] .
$$

Here the coupling constant is regularized by $g^{-1}=$ $M /(2 \pi a)-\sum_{\mathbf{k}}\left(2 \epsilon_{k}\right)^{-1}$, where $a$ is the s-wave scattering length, $M=m_{\uparrow} m_{\downarrow} /\left(m_{\uparrow}+m_{\downarrow}\right)$ is the reduced mass and $\epsilon_{k}=k^{2} / 2 M$.

Finally, we need an equation for $\Delta_{p g}^{2}$ which requires that we establish the dispersion of the non-condensed pairs. These non-condensed pairs, or pseudogap effects, appear at $T \neq 0$ and are included via a $T$-matrix contribution to the fermion self-energy. Following Refs. [7, 16], the fermionic self energy $\Sigma_{\sigma}(K)=\sum_{Q} t(Q) G_{\bar{\sigma}}(Q-K)$, where the four-momentum $Q=\left(i \Omega_{l}, \mathbf{q}\right), K=\left(i \omega_{n}, \mathbf{k}\right)$, and $\Omega_{l}\left(\omega_{n}\right)$ is the bosonic (fermionic) Matsubara frequency, with $\sum_{Q}=T \sum_{l} \sum_{\mathbf{q}}$, $\sum_{K}=T \sum_{n} \sum_{\mathbf{k}}$, and $\bar{\sigma}=-\sigma$. The $T$-matrix is presumed to have the structure $t(Q)=t_{s c}(Q)+t_{p g}(Q)$. The condensate contribution satisfies $t_{s c}(Q)=-\left(\Delta_{s c}^{2} / T\right) \delta(Q)$. Here the fermionic Green's function is $G_{\sigma}(K)=\left[G_{0 \sigma}^{-1}(K)-\right.$ $\left.\Sigma_{\sigma}(K)\right]^{-1}$, with $G_{0 \sigma}^{-1}(K)=\left(i \omega_{n}-\xi_{k \sigma}\right)$. We set $\hbar \equiv 1$ and $k_{B} \equiv 1$.

The excited pair propagator (which is what we need to arrive at our fourth equation) is given by $t_{p g}(Q)=\left[g^{-1}+\right.$ $\chi(Q)]^{-1}$, where the symmetrized pair susceptibility, $\chi(Q)=$ $\sum_{K, \sigma} G_{0 \sigma}(Q-K) G_{\bar{\sigma}}(K) / 2$, is used. A central assumption is the usual BEC condition that the pair chemical potential vanishes below $T_{c}$, which will, in turn, lead to Eq. (4). This

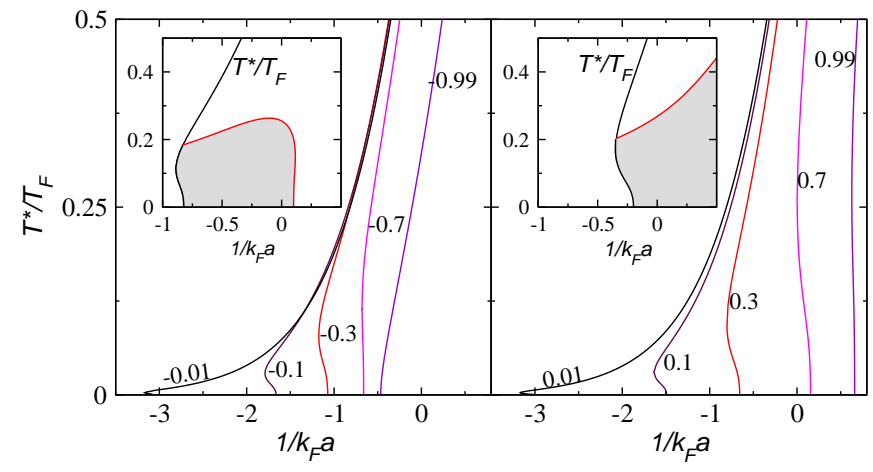

Figure 1: (Color online) $T^{*}$ as a function of $1 /\left(k_{F} a\right)$ for several values of $p$ (as labelled). Here $p<0$ when the lighter (spin up) species is the majority (left panel) and $p>0$ otherwise. Insets: $T^{*}$ (Black solid line) and unstable regime (shaded regime) for $p=-0.5$ and $p=0.5$, respectively.

BEC condition implies that $t_{p g}$ is dominated by terms with $Q \approx 0$. Importantly, the pseudogap is determined by $\Delta_{p g}^{2} \equiv$ $-\sum_{Q} t_{p g}(Q)$. It follows that $\Sigma_{\sigma}(K)=-\Delta^{2} G_{0 \bar{\sigma}}(-K)$, and in that way we have derived Eq. (1). One arrives at the two equations for the number densities via $n_{\sigma}=\sum_{K} G_{\sigma}(K)$ and in this way derives Eqs. (2) and (3).

The $T$-matrix may be expanded [17] as $t_{p g}^{-1}(Q)=a_{0} \Omega+$ $a_{1} \Omega^{2}-\xi^{2} q^{2}$, after analytic continuation $\left(i \Omega_{l} \rightarrow \Omega+i 0^{+}\right)$, where we have neglected the small imaginary part $\Gamma_{Q}$. The pseudogap contribution can be written as

$$
\Delta_{p g}^{2}=\sum_{\mathbf{q}} \frac{b\left(\tilde{\Omega}_{\mathbf{q}}\right)}{\sqrt{a_{0}^{2}+4 a_{1} \xi^{2} q^{2}}}
$$

Here $b(x)$ is the Bose distribution function and $\tilde{\Omega}_{\mathbf{q}}=$ $\left(\sqrt{a_{0}^{2}+4 a_{1} \xi^{2} q^{2}}-a_{0}\right) / 2 a_{1}$. In the BEC limit, it can be shown that $a_{1} / a_{0} \rightarrow 0$ and $\Omega_{\mathbf{q}} \rightarrow q^{2} / 2 M^{*}$, where $M^{*}=a_{0} / 2 \xi^{2}$ is the effective pair mass. Importantly, in this limit $M^{*}$ approaches the total mass of the two constituent fermions, and $T_{c}$ approaches the BEC temperature of ideal bosons of density $\min \left(n_{\uparrow}, n_{\downarrow}\right) / 2$ and mass $M^{*}$.

The gap equation, Eq. (4), is equivalent to an extremal condition on the thermodynamic potential $\partial \Omega_{M F} / \partial \Delta=0$, where

$\Omega_{M F}=-\frac{\Delta^{2}}{g}+\sum_{\mathbf{k}}\left(\xi_{k}^{+}-E_{k}\right)-T \sum_{\mathbf{k}, \sigma}\left[\ln \left(1+e^{-E_{k \sigma} / T}\right)\right]$.

Superfluid stability requires that the number susceptibility matrix $\partial n_{\sigma} / \partial \mu_{\sigma^{\prime}}$ should be positive-definite [7]. This can be shown to coincide with the condition that $\partial^{2} \Omega_{M F} / \partial \Delta^{2}>0$. When this condition is violated, phase separation may occur. These alternative phases have been widely studied [18] at $T=0$, as well as at non-zero temperature [19].

The distinction between $\Delta$ and the order parameter $\Delta_{s c}$ is an important component of the present theory. The former is associated with an onset temperature $T^{*}$ and the latter with 


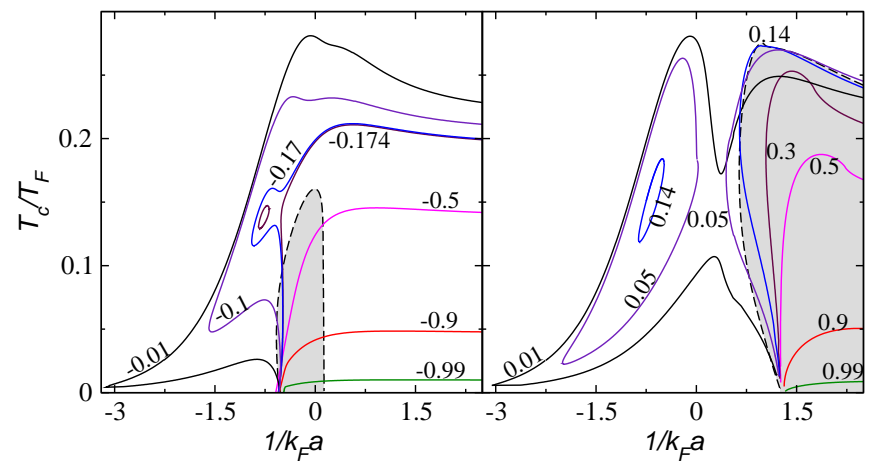

Figure 2: (Color online) $T_{c}$ as a function of $1 /\left(k_{F} a\right)$ for selected values of $p$. Here $p<0$ when the lighter species is the majority (left panel) and $p>0$ otherwise. The polarized superfluid solution is unstable in the shaded regions.

$T_{c}$. We arrive at a reasonable estimate of $T^{*}$ from Eqs. (2)(4) which is plotted in Fig. 1 as a function of $1 /\left(k_{F} a\right)$. Two different signs of the polarization $p=\delta n / n$ are indicated in the right and left panels. Here $k_{F}$ and $T_{F}$ are the Fermi momentum and the Fermi temperature of an unpolarized noninteracting Fermi gas with the same total particle density presuming a mass equal to the average mass of ${ }^{6} \mathrm{Li}$ and ${ }^{40} \mathrm{~K}$. We will see that throughout the paper a superfluid phase with $p<0$ (the lighter species is the majority) appears to be more readily obtainable than one with $p>0$. The arguments behind this asymmetry in $p$ are subtle and involve both energetic comparisons as well as mechanical stability (in the sense of $\partial^{2} \Omega_{M F} / \partial \Delta^{2}>0$ ). It can be analytically shown that at $T=0$ when polarization is small in the BEC regime, a superfluid phase with $p<0$ is more energetically favorable than one with $p>0$.

The figure shows that $T^{*}$ vanishes when the attraction is sufficiently weak and near its vanishing point it displays nonmonotonic behavior. Similar behavior has been observed previously for population imbalanced Fermi gases of equal masses [7]. The insets of Fig. 11indicate the unstable regimes for $p= \pm 0.5$. The unstable regimes are asymmetric in the sign of $p$ and at $T=0$ the behavior is consistent with phase diagrams obtained earlier [12, 13].

We turn next to the superfluid transition temperature, $T_{c}$, which is plotted in Fig. 2 as a function of $1 /\left(k_{F} a\right)$, for both $p<0$ (left panel) and $p>0$ (right panel). It is clear that these homogeneous superfluid phases are more likely to be observed when polarization is low. The shaded regions indicate where this form of superfluidity is unstable. Note that there is a rather pronounced asymmetry between the $p<0$ and $p>0$ cases. Indeed, when $p>0$, a stable superfluid cannot be found near $1 / k_{F} a=1.5$ although it will emerge again deep in BEC. An "intermediate temperature superfluid" phase exists in the BCS through unitary regimes, which is stable only away from the ground state. This is an unusual phase which has both a lower and an upper critical temperature, as previously found for population imbalanced same mass Fermi

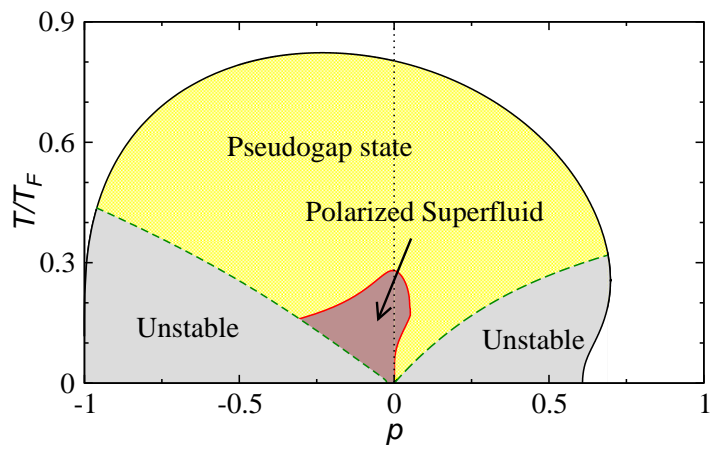

Figure 3: (Color online) Phase diagrams for mixtures of ${ }^{6} \mathrm{Li}$ and ${ }^{40} \mathrm{~K}$ atoms at unitarity. Here $p<0$ when ${ }^{6} \mathrm{Li}$ is the majority species and $p>0$ otherwise. The black solid, red solid, and green dashed lines are $T^{*}, T_{c}$, and the boundary of stable phases, respectively. Labeled are polarized superfluid (brown), pseudogap state (yellow) and unstable Sarma (grey) phases. The white open space is polarized normal Fermi gas.

gases [7].

Since the strongly interacting, unitary regime has been the focus of much interest, in Fig. 3 we first present the phase diagram at unitarity as a function of temperature and polarization $p$. It shows that uniformly polarized superfluid exists at low $|p|$ and low but finite $T$ (the dark shaded region). At higher $T$ and higher $|p|$, there is non-superfluid state with a finite excitation gap (called the pseudogap) before the system becomes essentially uncorrelated which we refer to as the normal Fermi gas state. However, at low $T$ and relatively high $|p|$, the Sarma superfluid phase (light shaded) is found to be unstable. Interestingly, when the heavier species is the majority (i.e., $p>0$ ), the stable or unstable paired states occupy a smaller phase space than the opposite case. It should be noted that when phase separation is included, it may cover the entire unstable region and extend slightly into the pseudogap and superfluid regions in the phase diagram. In this way the re-entrant behavior (seen upon a vertical cut a constant low $p$ ) shown in the right hand panel of Fig. 3 should not survive.

The boundary separating the pseudogap phase and the normal Fermi gas can be associated with $T^{*}$, while the line separating the polarized superfluid and the pseudogap phase corresponds to $T_{c}$. In a strict mean field calculation [13] there is no pseudogap so that the $T_{c}$ curve falls right on top of the $T^{*}$ line. It is clear, then, that pair fluctuation effects are extremely important for they greatly reduce the regime where superfluidity appears. In the equal mass case, where there is an opportunity to compare with experiments [20], a similar reduction in the regime of stable superfluid was found [21] which was in quite good agreement with the data. One can see from Fig. 3 that, for both $p<0$ and $p>0$, while mean-field theory again predicts a large value of the upper critical polarization (beyond which the superfluid phase cannot exist) and does not distinguish $T^{*}$ and $T_{c}$, our theory which includes pairing fluctuations predicts a smaller stable superfluid regime. Based on evidence in the equal-mass case, our prediction should be 


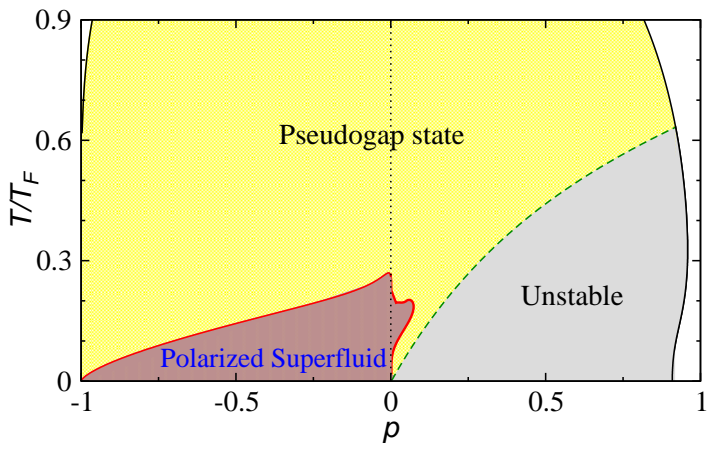

Figure 4: (Color online) Phase diagrams for mixtures of ${ }^{6} \mathrm{Li}$ and ${ }^{40} \mathrm{~K}$ atoms at $1 / k_{F} a=0.5$. The convention follows that in Fig. 3

more typical of future experiments.

Another notable feature of the figure is that when the heavier species is the majority, a stable homogeneously polarized superfluid only exists in a very narrow regime of extremely low polarization. This should serve as an important guide to future experiments, for it suggests one has to be very careful in order not to miss the (homogeneously) polarized superfluid in this case. It might seem as though the transition temperatures are much higher in the case of unequal masses than in the equal mass case. We stress that it is more meaningful to compare quantities such as $T_{c} / T^{*}$ than $T_{c} / T_{F}$ because, unlike in the equal mass case, here the energy unit $T_{F}$ does not correspond to the Fermi energy of ether species of the atoms. For the equal-mass case $T_{c} / T^{*} \approx 0.5$ while $T_{c} / T^{*} \approx 0.3$ for a mixture of ${ }^{6} \mathrm{Li}$ and ${ }^{40} \mathrm{~K}$ atoms at $p=0$.

To address how the phase diagram evolves from unitarity to the BEC side of the Feshbach resonance, we present in Fig. 4 the counterpart phase diagrams at $1 / k_{F} a=0.5$ for the interspecies superfluid. It should be clear from the figure that, just as in the previous case, pair fluctuation effects which allow us (via Eq. (1)) to distinguish between the gap $\Delta$ and the order parameter $\Delta_{s c}$, are extremely important as they greatly reduce the regime of stable homogeneous superfluidity.

When the heavier species is the majority $(p>0)$, there is virtually no stable polarized superfluid. Again, the re-entrant behavior of the intermediate temperature superfluid at low $p>0$ in Fig. 44should not survive if phase separation is considered. In contrast, when the lighter species is the majority $(p<0)$, there is no longer an intermediate temperature superfluid phase. Rather a stable superfluid can be found for all polarizations and temperatures below the (single) critical temperature $T_{c}$. This behavior can be contrasted with the equal mass case where, for, e.g., $p=0.5$ at $T=0$, a stable superfluid cannot be found until deep in the BEC regime, when $1 / k_{F} a>2$. For a moderate polarization, say $p=-0.5$, this transition temperature $T_{c} \approx 0.18 T_{F}$, which should be accessible in future.

Although we have considered the homogeneous rather than a trapped case, there are now experimental capabilities for addressing this phase diagram using tomography [22]. More- over, we have previously [21] characterized the changes in the phase diagram for the equal mass case upon going from the homogeneous to the trapped situation. We have shown that, while the regimes of stability of generalized Sarma phases are greatly expanded in a trap, the characteristic values of the transition temperatures are not significantly altered.

This work is supported by NSF PHY-0555325 and NSFMRSEC Grant 0820054. We thank Cheng Chin for helpful conversations.

[1] M. W. Zwierlein, A. Schirotzek, C. H. Schunck, and W. Ketterle, Science 311, 492 (2006).

[2] Y. Shin, M. W. Zwierlein, C. H. Schunck, A. Schirotzek, and W. Ketterle, Phys. Rev. Lett. 97, 030401 (2006).

[3] G. B. Partridge, W. Li, R. I. Kamar, Y. A. Liao, and R. G. Hulet, Science 311, 503 (2006).

[4] G. B. Partridge, W. Li, Y. A. Liao, R. G. Hulet, M. Haque, and H. T. C. Stoof, Phys. Rev. Lett. 97, 190407 (2006).

[5] C. H. Schunck, Y. Shin, A. Schirotzek, M. W. Zwierlein, and W. Ketterle, Science 316, 867 (2007).

[6] G. Sarma, J. Phys. Chem. Solids 24, 1029 (1963).

[7] C. C. Chien, Q. J. Chen, Y. He, and K. Levin, Phys. Rev. Lett. 97, 090402 (2006); Q. J. Chen, Y. He, C. C. Chien, and K. Levin, Phys. Rev. A 74, 063603 (2006).

[8] P. F. Bedaque, H. Caldas, and G. Rupak, Phys. Rev. Lett. 91, 247002 (2003); H. Caldas, Phys. Rev. A 69, 063602 (2004).

[9] E. Wille, F. M. Spiegelhalder, G. Kerner, D. Naik, A. Trenkwalder, G. Hendl, F. Schreck, R. Grimm, T. G. Tiecke, J. T. M. Walraven, et al., Phys. Rev. Lett. 100, 053201 (2008).

[10] G. Nardulli, in Ultra-cold Fermi Gases, edited by M. Inguscio, W. Ketterle, and C. Salomon (Italian physical society, Bologna, 2007), p. 797.

[11] S. Pilati and S. Giorgini, Phys. Rev. Lett. 100, 030401 (2008).

[12] M. Iskin and C. A. R. Sa de Melo, Phys. Rev. Lett. 97, 100404 (2006); M. Iskin and C. A. R. Sa de Melo, Phys. Rev. A 76, 013601 (2007); M. Iskin and C. A. R. Sa de Melo, Phys. Rev. A 77, 013625 (2008).

[13] M. M. Parish, F. M. Marchetti, A. Lamacraft, and B. D. Simons, Phys. Rev. Lett. 98, 160402 (2007).

[14] S. T. Wu and C. H. Pao, Phys. Rev. B 74, 224504 (2006); C. H. Pao, S. T. Wu, and S. K. Yip, Phys. Rev. A 76, 053621 (2007).

[15] W. Yi and L. M. Duan, Phys. Rev. A 73, 031604(R) (2006).

[16] Q. J. Chen, J. Stajic, S. N. Tan, and K. Levin, Phys. Rep. 412, 1 (2005).

[17] In contrast to previous work we take higher terms $\left(a_{1} \Omega^{2}\right)$ in the T-matrix expansion to avoid numerical complications from vanishing $a_{0}$.

[18] D. E. Sheehy and L. Radzihovsky, Phys. Rev. Lett. 96, 060401 (2006).

[19] C.-C. Chien, Q. J. Chen, Y. He, and K. Levin, Phys. Rev. Lett. 98, 110404 (2007).

[20] Y. I. Shin, C. H. Schunck, A. Schirotzek, and W. Ketterle, Nature 451, 689 (2008).

[21] Q. Chen, Y. He, C. C. Chien, and K. Levin, eprint, arXiv:0810.1940

[22] A. Schirotzek, Y. Shin, C. H. Schunck, and W. Ketterle, Phys. Rev. Lett. 101, 140403 (2008). 